\title{
DESCRIPCIÓN DEL INTENTO SUICIDA EN ARMENIA 2016-2018.
}

\author{
SUICIDE ATTEMPT IN ARMENIA 2016-2018 DESCRIPTION
}

Carmen Luisa Betancur-Pulgarin ${ }^{1}$ (D) ; María del Pilar Marín-Giraldo ${ }^{2}$ (D) ; Juliana Escobar-Angel ${ }^{3 *}$ (iD); Diana Karina Marín-Rodríguez ${ }^{4}$ (iD); Laura Isabella Ortega-Muñoz ${ }^{5}$ (D).

1. Profesora y enfermera docente asociada Fundación universitaria del Área Andina-Pereira. Magister en enfermería, especialista en epidemiología y docencia universitaria.

2. Magister en Salud Pública, especialista en epidemiología, enfermera y docente asociada de la Universidad Católica de Manizales y Fundación Universitaria del Área Andina-Pereira.

3. Médica de la Fundación Cardiomet Cequin-Armenia, estudiante de Epidemiología.

4. Médica del Hospital La misericordia-Calarca, estudiante de Epidemiología.

5. Médica de Americares ONG-Cali, estudiante de Epidemiología.

*Correspondencia del Autor: Juliana Escobar-Angel, correo electrónico: jescobar61@estudiantes.areaneandina.edu.co

Dirección: Parque residencial los ocobos; Calle 31 norte \# 20-10, casa 5 manzana.

\section{RESUMEN}

Introducción: La incidencia en Colombia del intento de suicidio ha ido en aumento año tras año, siendo el Quindío uno de los departamentos con una tasa de incidencia superior a la del país por lo que se hace necesario la caracterización de la población y la identificación de los factores relacionados con el intento de suicidio en la región. Objetivo: Describir el intento de suicidio en la ciudad de Armenia durante el periodo $2016-2018$.

Metodología: Se realizó un estudio observacional descriptivo con un primer intento suicida notificado por el SIVIGILA, se utilizó estadística descriptiva, pruebas de Chi cuadrado y Mann Whitney.

Resultados: Se analizó una muestra de 598 casos notificados de intento de suicidio donde más del 50\% fueron menores de 29 años y según su estado civil el 68,9\% se encontraba sin pareja; el estrato socioeconómico predomínate fue bajo, el mecanismo utilizado más frecuente fue la intoxicación. Se encontró relación entre intentos de suicidios previos y enfermedad crónica, problemas jurídicos, antecedente de trastorno psiquiátrico y consumo de SPA. Según el periodo epidemiológico en los años analizados la tendencia fue creciente para los periodos IX y XI.

Palabras clave: Suicidio; intento de suicidio; ideación suicida; conducta autodestructiva; factores de riesgo; factores precipitantes; MeSH.

Cómo citar:

Betancur-Pulgarin, Carmen Luisa; Marín-Giraldo, María del Pilar; Escobar-Angel, Juliana; Marín-Rodríguez, Diana Karina; Ortega-Muñoz, Laura Isabella. (2020). Descripción del intento suicida en Armenia 2016-2018. Revista de Investigaciones Universidad del Quindio, 32(1), 79-89. https://doi.org/10.33975/riuq.vol32n1.285

Información del artículo: Recibido: 17 abril 2020; Aceptado: 30 agosto 2020

Revista de Investigaciones Universidad del Quindío,

32(1), 79-89; 2020.

ISSN: 1794-631X e-ISSN: 2500-5782

Esta obra está bajo una licencia Creative Commons Atribución- 


\section{ABSTRACT}

Introduction: The national incidence rate of the suicide attempts has grown year after year, where the department of Quindío is above the countries' rate, making it mandatory to have a characterization of the population as well as the identification of the factors related to the attempt of suicide in the region.

Objective: Describe the attempt of suicide in the city of Armenia (Quindío) between 2016 and 2018.

Methodology: A descriptive observational study was conducted with a first suicide attempted reported by SIVIGILA using descriptive statistics, Chi square tests and Mann Whitney.

Results: A sample of 598 reported suicide attempted cases was analyzed, where more of the $50 \%$ were people under 29 years old, and according to their marital status, $68.9 \%$ did not have a partner at the moment, the predominant socioeconomic stratum was low and the most frequent mechanism was poisoning. There was a finding in the relation between previous suicide attempts and legal problems, psychiatric disorder backgrounds and consumption of psychoactive substances (SPA). According to the epidemiological period in the years analyzed, the tendency was increasing for the IX and XI periods.

Key Words: Suicide; suicide attempted; suicidal Ideation; risk factors; Precipitating Factor; MeSH.

\section{INTRODUCCIÓN}

Según la Organización mundial de la salud, más de 800.000 personas se suicidan por año, por cada uno de los suicidios se calculan 20 intentos, la población juvenil es la más afectada, siendo la segunda causa principal de defunción en el grupo de 15 a 29 años en todo el mundo (1)age group, year, region and/ or department from 2000 to 2013. The databases of deaths by suicide and population projections of DANE were used. A longitudinal and analytical study was conducted. From 2000 to 2013, annual trends of deaths by suicide by means of modeling of covariables were established and risk estimates were collected. The Poisson regression model (PRM. Según el estudio de Rawat en el 2019 se ratifica que los hombres se suicidan en mayor proporción que las mujeres sin embargo las mujeres realizan más intentos que los hombres; se utiliza más, el método no violento en ambos grupos pero el método violento es representativo en las personas que se suicidan (2). En América ocurren aproximadamente 65.000 defunciones anuales por suicidio y una tasa de mortalidad ajustada según la edad de 7,3 por 100.000 habitantes en el periodo 2005-2009, según la Organización Panamericana de la salud. (3).

El estudio de cohorte de Asarnow en el 2017 es uno de los estudios que ha demostrado que haber sobrevivido a un intento suicida es uno de los más robustos predictores de muerte suicida, en dicho estudio se encontró que, ante la combinación de un intento o comportamiento suicida previo, la depresión y el consumo de alcohol conforman una triada que se asocia con una probabilidad significativamente mayor de comportamiento suicida (4). En otro estudio transversal de LópezGoñi JJ en el 2018 describe que 2/3 de las muertes por suicidio se producen en un primer intento $\mathrm{y}$ para los que tienen un próximo intento tienen una probabilidad mayor (40-66\%) de morir por suicidio que la población general (5). El suicidio y las conductas asociadas se han convertido en una de las causas crecientes de demanda de asistencia sanitaria, aproximadamente $1 / 3$ de los pacientes atendidos en urgencias psiquiátricas han tenido 
conductas suicidas generando altos costos en el manejo, seguimiento y cuidado de las secuelas o discapacidades como lo demuestra el estudio de Jiménez Q M del 2014 (6).

Se observó al igual que en Latinoamérica, que en países orientales el suicidio es un grave problema de salud pública, de tal forma que en esta región se hizo obligatorio realizar investigación sobre las causales y características del fenómeno en la población, desde el método seleccionado hasta los parámetros demográficos y mentales de la población (7). En los países subdesarrollados se ha observado mayor prevalencia del suicidio en donde la razón hombre a mujer se ha calculado en 1,5 suicidios masculinos por cada suicidio femenino, donde ocurren anualmente cerca de 65.000 defunciones por suicidio asociados (8). En México Los factores de riesgo de suicidio más recurrentes en el país son el consumo nocivo de alcohol o drogas, dolores y enfermedades crónicas, desórdenes psiquiátricos y eventos negativos en la vida (9).

En Colombia se ha presentado un aumento progresivo de la tasa de suicidio en los últimos años, pasando de 4,5 por cada 100.000 habitantes para el año 2009, a 5,93 por cada 100.000 habitantes en el año 2018, siendo este año el que registra mayor número de suicidios, cuya mayor población son personas entre 20 a 39 años de edad, en su mayoría hombres (10). Además entre el 2000 y 2010, se encontró una mayor prevalencia de suicidio en los adultos jóvenes (9,0/100.000 habitantes) relacionado con su ciclo de vida, el consumo de sustancias psicoactivas y problemas sociales, los adultos mayores se encontraron con una tasa de 6,9/100.000 habitantes, relacionada con la jubilación, abandono, pérdida de la pareja (11).

Por otra parte, en Colombia el $80 \%$ de las personas que mueren por suicidio tienen una enfermedad mental de base, sin embargo, el 98\% de las personas con trastornos mentales no mueren por suicidio y más del $30 \%$ de la población que intentó suicidarse en 2016 ya lo había intentado antes donde la ideación suicida persistente fue el factor asociado con 2 o más intentos (12). Para el 2003 al menos el 4,9\% de la población había tenido un intento suicida, el 12,3\% había tenido ideación suicida y el 4,1 $\%$ planearon suicidio; el principal predictor de conducta suicida se asoció a depresión, intento suicida previo y antecedente familiar de suicidio; los antecedentes de enfermedad psiquiátrica más relacionados a conducta suicida son la depresión y el trastorno afectivo bipolar; los conflictos familiares que juegan un papel importante como desencadenantes del intento suicida, ya sea por separación de los padres o el maltrato familiar (13).

Para el 2015 se encontró una mayor prevalencia de intento suicida en mujeres, no se evidencia diferencias entre adultos en situación de pobreza y quienes no lo son, en la escolaridad se evidencia mayor ideación suicida entre las personas con secundaria completa, hay mayor proporción de ideación en personas separadas viudas y divorciadas, mayor ideación suicida en personas con depresión, trastornos de ansiedad y personalidad limítrofe (14). En 2018, se concluyó que la conducta suicida en la población colombiana predomina el siguiente perfil: hombres, jóvenes de 20 a 39 años de edad, con educación básica, solteros, residentes en la cabeceras municipales y con enfermedad física o mental, tener antecedentes familiares de suicidio, intentos previos, contar con una mala salud física, tener armas de fuego en casa o padecer una enfermedad mental (15).

El departamento del Quindío se encuentra de tercero entre los cinco departamentos con mayor tasa de suicidio a nivel nacional (dentro de las mayores tasas se incluye el departamento de Risaralda y caldas constituyendo así una zona de importancia el Eje cafetero) donde, en el 2015 se presentó una tasa de suicidio en el Quindío de 7.5 por 100.000 habitantes, en el 2018 la tasa aumentó a 10.7 por 100.000 habitantes, siendo el municipio de armenia uno de los más afectados, donde se observó que tener una unión marital 
puede disminuir 50\% la posibilidad del suicidio; por otro lado se identificó un aumento de los eventos entre 1999-2003 en el cual se sugirió una posible relación con el sismo del 25 enero de 1999 que afectó en gran medida al eje cafetero en especial al territorio Quindiano (16).

Para explicar el espectro de la conducta asociada al suicidio, se abarca desde la ideación o planificación de actos voluntarios e intencionales que tengan el fin de producirse daño corporal como respuesta a la problemática por la cual pasa en dicho momento, no obstante aún no se ha producido un daño físico contra sí mismo, de modo que el intento suicida es donde se llevan a cabo dichas actividades donde el resultado final no es la muerte pero se tiene como consecuencias el dolor, la desfiguración o el daño de alguna función o parte del cuerpo, sin embargo se puede terminar consumando éste teniendo como resultado el suicidio (17). En relación con el ser humano se debe entender el término de salud mental; la OMS define la salud como un estado de completo bienestar físico, mental y social, no solamente la ausencia de afecciones o enfermedades (18). Actualmente, se plantean 2 teorías: la motivacional-volitiva e interpersonal, en la última, Thomas Joiner relaciona factores integrados por la teoría del modelo integral motivacional como el fracaso, el atrapamiento personal, el grado de apoyo social, y en su fase volitiva, la perdida al temor de la muerte y la impulsividad, aun sin lograr darle explicación al fenómeno; en un estudio de análisis de redes de estos componentes con respecto a la ideación suicida el $16 \%$ de la ideación es explicada por la teoría interpersonal y $23 \%$ por la teoría motivacional- volitiva (19).

En relación a los factores de riesgo se ha encontrado que la depresión puede incrementar hasta 12 veces el riesgo de intento de suicidio cuando predomina la desesperanza, asociada con intencionalidad (20). El intento suicida está fuertemente asociado a trastornos mentales por lo que se hace necesario comprender mejor los procesos que motivan y conducen a pensamientos suicidas y el por qué algunas personas cruzan el umbral para llevar a cabo el acto suicida (21). Las personas con riesgo suicida presentan manifestaciones prodrómicas y el principal predictor de muerte por esta causa es un intento previo, durante los seis primeros meses e incluso durante el primer año el riesgo aumenta entre 2030 veces (22).

Las estrategias enfocadas en el seguimiento de aquellos pacientes con intento suicida, en especial en los meses siguientes al evento, resaltan programas de seguimiento telefónico que al implementarlas reducen la reincidencia (23); como factores protectores de suicidio en esta población se puede resaltar el mejoramiento de las habilidades sociales, la participación y aceptación activa de la familia (24), y la intervención interdisciplinaria de trabajo social, psicología y psiquiatría; para Colombia la OMS describe una proporción de 2,5 psiquiatras por 100.000 individuos de tal manera que suele retrasarse la atención (25).

Según la Guía de práctica clínica del intento suicida del Ministerio Colombiano de Salud, se debe realizar una adecuada evaluación psicopatológica y social que incluya las características psicológicas, así como una evaluación de los factores de riesgo (26). Las personas cercanas o expuestas a este suceso como familiares, amigos o vecinos de las víctimas podrían repetir este evento, lo que hace necesario delimitar geográficamente regiones de ocurrencia y evitar replicación de los mismos (27). Entonces las acciones individuales deben contemplar la alerta a la EPS de cada caso identificado, para promover la intervención oportuna y el seguimiento (28).

Teniendo en cuenta lo anterior se hace necesaria la caracterización de la población, la identificación de las condiciones causales y de atención en el intento de suicidio, donde el principal factor de riesgo son los intentos previos, de tal manera que este estudio busca describir el intento suicida de la población de Armenia durante el periodo 2016- 
2018, además de la ocurrencia de estos según los periodos epidemiológicos.

\section{METODOLOGÍA}

Se realizó un estudio observacional descriptivo, en la población total (598 casos notificados) de personas mayores de 18 años con un primer intento suicida entre 2016-2018 por la ficha del SIVIGILA en la ciudad de Armenia, se obtuvo la información a partir de la base de datos anonimizada suministrada por la Secretaría de Salud de Armenia de la notificación individual de intento suicida del SIVIGILA. La tabulación de los datos se construyó en una base de datos en Microsoft Excel Licenciado, la cual fue exportada al software EPI INFO 7.2 para posterior análisis con estadística descriptiva, medidas de tendencia central, de dispersión y posición, para analizar normalidad se aplicó prueba de Kolmogorov Smirnow. Para el análisis bivariado se utilizaron pruebas de Chi cuadrado y Mann Whitney.

En algunas variables no se tiene información en las unidades de análisis ya que dicha información proviene de las fichas de notificación de intento de suicidio del SIVIGILA las cuales han sufrido modificaciones y en las últimas versiones se han incluido nuevas variables por lo que se codifica sin dato.

Para el análisis bivariado se agrupó algunas de las categorías como: Estado civil: con pareja y sin pareja; escolaridad: básico o ninguno y medio/alta; pertenencia étnica: pertenece y no pertenece; tipo de régimen en salud: asegurado y no asegurado; área de ocurrencia: urbano y rural; se agrupó las siguientes opciones en caso de antecedente psiquiátrico, trastorno depresivo, trastorno de personalidad, trastorno bipolar y esquizofrenia, y se marcó con Si los que cumplían alguna de esas variables, y como No los que no la cumplían. No se tuvo en cuenta la variable estrato pues cuenta con más del 50\% de unidad de análisis sin dato, de la misma forma no se pudo analizar los intentos previos con los grupos poblacionales: discapacitados, migrantes y víctimas de violencia ya que la ocurrencia del evento se da o no en el $0 \%$ y $100 \%$ de esta población.

Este estudio cuenta con la aprobación del Comité de Ética en Investigación de la Universidad Católica de Oriente del 12 de diciembre de 2019 que consta en el acta $\mathrm{N}^{\circ} 22$ ordinaria y se realizó cumpliendo la norma ISO 26000.

\section{RESULTADOS}

Se encontró que por cada 100 intentos de suicidio en mujeres se presentan 83 intentos de suicidio en hombres, más del $50 \%$ de la población son menores de 29 años, con una edad máxima de 89 años, el 4\% ocurre en área rural, el 0,5\% pertenece a una etnia (Rom, gitano o raizal) y el $84,9 \%$ cuenta con un aseguramiento en el régimen de salud. El 68,9\% (IC 64,3 a 73,3) de la población con la que se contaba con información $(n=403)$ se encontraba sin pareja; el 75,7\% (IC $70,8$ a 80$)$ de la población $(n=334)$ tenían un nivel de escolaridad básico o ninguno. El 93,8\% de la población $(n=178)$ pertenece a un estrato socioeconómico bajo (considerados estratos 1,2 y 3 ), con una pérdida de dato del 70,2\%. Los grupos poblacionales representativos fueron los provenientes de centros psiquiátricos y centros carcelarios con un porcentaje del 6,19\% y 1,67\% respectivamente. Además por cada 100 personas que intentaron suicidarse y no tenían intentos previos 48 si tenían intentos previos, donde el $18,2 \%$ tienen 2 o más intentos previos. Los factores desencadenantes más frecuentes son el conflicto con pareja o expareja, problemas económicos, enfermedad crónica dolorosa o discapacitante y problemas jurídicos, el 39\% no contaban con factores desencadenantes y el 12,04\% contaban con dos o más factores. Los factores de riesgo más predominantes fueron: consumo de SPA, ideación suicida persistente y antecedente de algún trastorno psiquiátrico estando éste presente en el 39,8\% de la población. El mecanismo más utilizado fue la intoxicación con un $70 \%$ de la muestra y se reportó remisiones en el 95\% de la población para el servicio de psiquiatría. (Ver Tabla 1). 
Tabla 1. Distribución de características sociodemográficas, grupos poblacionales, intentos suicidas previos, factores desencadenantes, factores de riesgo, mecanismo y especialidad a la que se remite en intento suicida en Armenia, 20162018.

\begin{tabular}{|c|c|c|c|}
\hline Variables & Categoría & Frecuencia absoluta & Frecuencia relativa \\
\hline \multirow{2}{*}{ Sexo } & Femenino & 326 & $54,52 \%$ \\
\hline & Masculino & 272 & $45,48 \%$ \\
\hline \multicolumn{2}{|c|}{ Edad en años* } & \multicolumn{2}{|c|}{$28 \pm 16$} \\
\hline \multirow{6}{*}{ Estado civil } & Soltero & 254 & $42,47 \%$ \\
\hline & Sin dato & 195 & $32,61 \%$ \\
\hline & Casado & 65 & $10,87 \%$ \\
\hline & Unión libre & 60 & $10,03 \%$ \\
\hline & Viudo & 15 & $2,51 \%$ \\
\hline & Divorciado & 9 & $1,51 \%$ \\
\hline \multirow{14}{*}{ Escolaridad } & Preescolar & 12 & $2,01 \%$ \\
\hline & Básica primaria & 101 & $16,89 \%$ \\
\hline & Básica secundaria & 137 & $22,91 \%$ \\
\hline & Media técnica & 20 & $3,34 \%$ \\
\hline & Técnica profesional & 11 & $1,84 \%$ \\
\hline & Tecnológica o técnica & 10 & $1,67 \%$ \\
\hline & Profesional & 38 & $6,35 \%$ \\
\hline & Especialización & 2 & $0,33 \%$ \\
\hline & Maestría & 0 & $0 \%$ \\
\hline & Doctorado & 0 & $0 \%$ \\
\hline & Sin información & 50 & $8,36 \%$ \\
\hline & Ninguno & 3 & $0,50 \%$ \\
\hline & Sin dato & 195 & $32,61 \%$ \\
\hline & 4 (No especificado) & 19 & $3,18 \%$ \\
\hline \multirow{7}{*}{$\begin{array}{l}\text { Estrato socio- } \\
\text { económico }\end{array}$} & 1 & 30 & $5,02 \%$ \\
\hline & 2 & 94 & $15,72 \%$ \\
\hline & 3 & 43 & $7,19 \%$ \\
\hline & 4 & 4 & $0,67 \%$ \\
\hline & 5 & 3 & $0,50 \%$ \\
\hline & 6 & 4 & $0,67 \%$ \\
\hline & Sin dato & 420 & $70,23 \%$ \\
\hline \multirow{6}{*}{ Régimen de salud } & Contributivo & 267 & $44,65 \%$ \\
\hline & Subsidiado & 217 & $36,29 \%$ \\
\hline & No asegurado & 85 & $14,21 \%$ \\
\hline & Excepción & 19 & $3,18 \%$ \\
\hline & Especial & 5 & $0,84 \%$ \\
\hline & Indeterminado/pendiente & 5 & $0,84 \%$ \\
\hline \multirow{3}{*}{ Pertenencia étnica } & Otros & 595 & $99,50 \%$ \\
\hline & Rom, gitano & 2 & $0,33 \%$ \\
\hline & Raizal & 1 & $0,17 \%$ \\
\hline
\end{tabular}




\begin{tabular}{|c|c|c|c|}
\hline \multirow{3}{*}{ Área de ocurrencia } & Cabecera municipal & 569 & $95,15 \%$ \\
\hline & Rural disperso & 26 & $4,35 \%$ \\
\hline & Centro poblado & 3 & $0,50 \%$ \\
\hline \multicolumn{4}{|c|}{${ }^{*}$ Los datos se presentan en mediana \pm Rango intercuartil } \\
\hline \multirow{9}{*}{$\begin{array}{l}\text { Factores desencade- } \\
\text { nantes }\end{array}$} & Conflictos con pareja o expareja & 230 & $38,46 \%$ \\
\hline & $\begin{array}{c}\text { Enfermedad crónica dolorosa o } \\
\text { discapacitante }\end{array}$ & 55 & $9,20 \%$ \\
\hline & Problemas económicos & 55 & $9,20 \%$ \\
\hline & Muerte de un familiar & 64 & $10,70 \%$ \\
\hline & Escolar/ educativo & 12 & $2,01 \%$ \\
\hline & Problemas jurídicos & 49 & $8,19 \%$ \\
\hline & Suicidio de un familiar o amigo & 2 & $0,33 \%$ \\
\hline & Problemas laborales & 24 & $4,01 \%$ \\
\hline & $\begin{array}{c}\text { Maltrato físico, psicológico o } \\
\text { sexual }\end{array}$ & 10 & $1,67 \%$ \\
\hline \multirow{7}{*}{ Factores de riesgo } & Consumo de spa & 129 & $21,57 \%$ \\
\hline & Antecedente de conducta suicida & 18 & $3,01 \%$ \\
\hline & Ideación suicida persistente & 130 & $21,74 \%$ \\
\hline & Plan organizado de suicidio & 18 & $3,01 \%$ \\
\hline & Abuso de alcohol & 32 & $5,35 \%$ \\
\hline & Antecedente de violencia & 397 & $66,39 \%$ \\
\hline & Trastorno psiquiátrico & 158 & $26,42 \%$ \\
\hline
\end{tabular}

Según el periodo epidemiológico en los años analizados la tendencia fue creciente para los periodos IX y XII. Con un amento de ocurrencia para el año 2018. (Ver Gráfica 1).

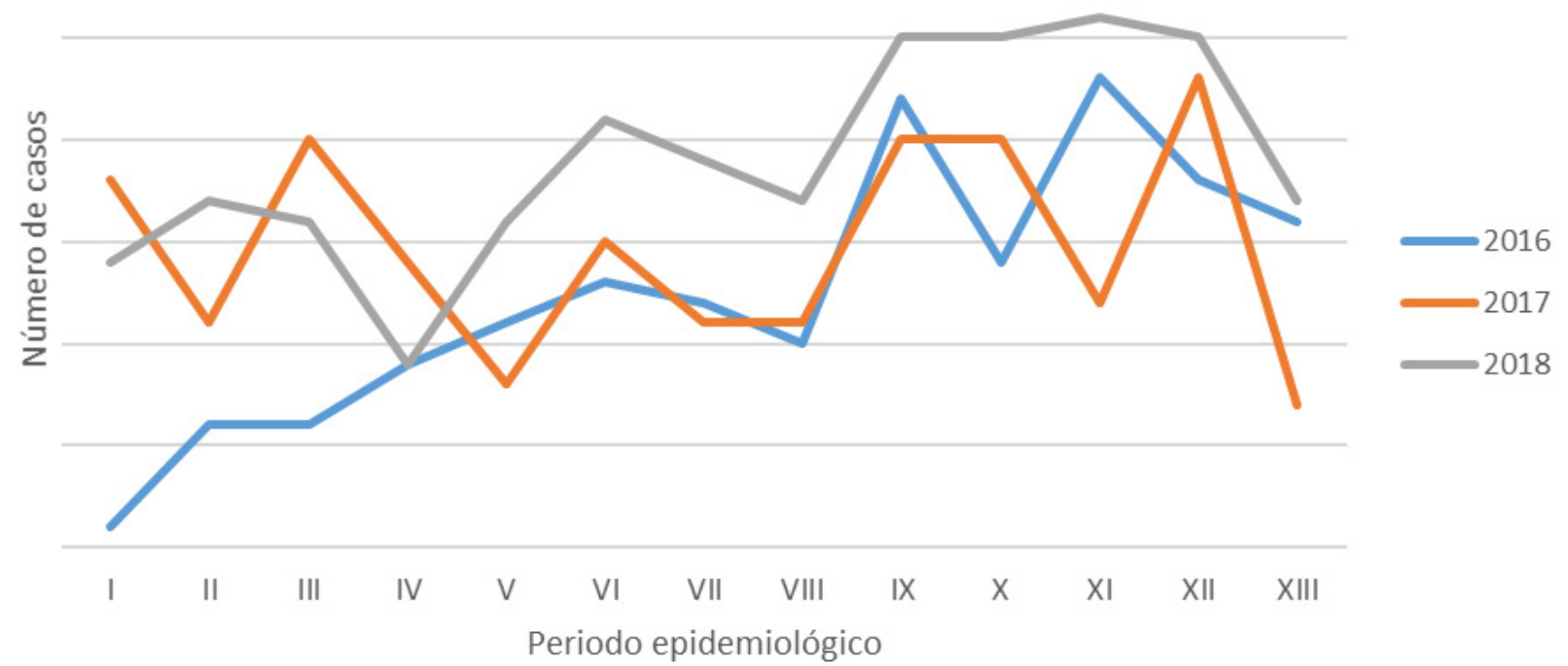

Gráfica 1. Distribución por años y periodos epidemiológicos del intento suicida, Armenia, 2016-2018. 
Los intentos de suicidio previos se relacionaron con una mediana de edad de 26 años $(\mathrm{P}<0,001)$, hay una relación entre el estado civil y los intentos previos: estar sin pareja es un factor de riesgo $(p=0,02)$. En cuanto a los grupos poblacionales, se encontró relación entre las personas pertenecientes a centros psiquiátricos y los intentos previos de suicidio $(p<0,001)$, además se encontró relación entre la presencia de enfermedad crónica dolorosa o discapacitante y los intentos previos, así como tener problemas jurídicos $(\mathrm{p}<0,001)$. Se encontró relación entre consumo de sustancias psicoactivas $(\mathrm{P}=0,003)$, ideación suicida persistente $(\mathrm{p}<0,001)$, antecedente de algún trastorno psiquiátrico $(\mathrm{p}<0,001)$. (Ver Tabla 2).

Tabla 2. Relación entre intentos previos y características sociodemográficas, grupos poblacionales, factores desencadenantes, factores de riesgo, Armenia 2016-2018.

\begin{tabular}{|c|c|c|c|c|c|c|}
\hline & & \multicolumn{2}{|c|}{ Intentos previos } & Valor $p$ & OR & IC 95\% \\
\hline Variable & Categorías & SI n(\%) & NO n(\%) & & & \\
\hline \multicolumn{2}{|c|}{ Edad en años* } & $26(21 ; 36)$ & $28(22 ; 38)$ & $<0,001$ & N.A & N.A \\
\hline \multirow[t]{2}{*}{ Estado civil } & Sin pareja & 37,41 & 62,59 & \multirow[t]{2}{*}{0,02} & \multirow[t]{2}{*}{1,73} & \multirow[t]{2}{*}{1,09 a 2,78} \\
\hline & Con pareja & 25,6 & 74,4 & & & \\
\hline \multicolumn{7}{|c|}{ Grupos Poblacionales } \\
\hline \multirow[t]{2}{*}{ Centro psiquiátrico } & $\mathrm{Si}$ & 62,16 & 37,84 & \multirow[b]{2}{*}{$<0,001$} & \multirow[b]{2}{*}{3,7} & \multirow[t]{2}{*}{1,87 a 7,39} \\
\hline & No & 30,66 & 6,34 & & & \\
\hline \multicolumn{7}{|c|}{ Factores desencadenantes } \\
\hline \multirow{2}{*}{$\begin{array}{c}\text { Enfermedad crónica } \\
\text { dolorosa }\end{array}$} & $\mathrm{Si}$ & 56,36 & 43,64 & \multirow[b]{2}{*}{$<0,001$} & \multirow[b]{2}{*}{2,98} & \multirow[t]{2}{*}{1,70 a 5,24} \\
\hline & No & 30,2 & 69,8 & & & \\
\hline \multirow[t]{2}{*}{ Problemas jurídicos } & $\mathrm{Si}$ & 46,94 & 53,06 & \multirow[t]{2}{*}{0,02} & \multirow[t]{2}{*}{1,94} & \multirow[t]{2}{*}{1,07 a 3,49} \\
\hline & No & 31,33 & 68,67 & & & \\
\hline \multicolumn{7}{|c|}{ Factores de riesgo } \\
\hline \multirow[t]{2}{*}{ Consumo SPA } & $\mathrm{Si}$ & 43,41 & 56,59 & \multirow[t]{2}{*}{0,003} & \multirow[t]{2}{*}{1,8} & \multirow[t]{2}{*}{1,2 a 2,7} \\
\hline & No & 29,64 & 70,36 & & & \\
\hline \multirow{2}{*}{$\begin{array}{c}\text { Ideación Suicida per- } \\
\text { sistente }\end{array}$} & $\mathrm{Si}$ & 61,54 & 38,46 & \multirow[t]{2}{*}{$<0,001$} & \multirow[t]{2}{*}{4,9} & \multirow[b]{2}{*}{3,25 a 7,41} \\
\hline & No & 24,57 & 75,43 & & & \\
\hline \multirow{2}{*}{$\begin{array}{l}\text { Antecedente de } \\
\text { trastorno psiquiátrico }\end{array}$} & $\mathrm{Si}$ & 46,22 & 53,78 & \multirow[b]{2}{*}{$<0,001$} & \multirow[b]{2}{*}{2,78} & \multirow[b]{2}{*}{1,95 a 3,5} \\
\hline & No & 23,61 & 76,39 & & & \\
\hline
\end{tabular}

\section{DISCUSIÓN}

Los resultados del estudio permiten acercarse al entendimiento del comportamiento del intento suicida en la ciudad de Armenia, según las características sociodemográficas se encontró una ligera diferencia porcentual entre hombres y mujeres con reporte de intento suicida, siendo predominante el sexo femenino, similar a lo encontrado en la literatura donde describen que los hombres se suicidan en mayor proporción que las mujeres sin embargo las mujeres realizan más intentos suicidas que los hombres (2). Sin embargo cabe resaltar que la asociación epidemiológica con el intento previo no fue estadísticamente significativa; se encontró igualmente una mediana de edad de 28 años coherente con el rango de presentación en Colombia según el estudio Forensis de 2018 con edades comprendidas entre 20 y 39 años, siendo para este estudio el $78 \%$ en este rango de edad (29).

Para las variables sexo, área de ocurrencia, régimen de salud, y grupo étnico no se encontró relación comparada con intentos previos. A diferencia del estrato socioeconómico bajo 
(estrato 1,2 y 3 ) el cual fue el de mayor proporción en nuestra población que difiere del estudio de referencia (30). Respecto al estado civil las personas sin pareja fueron las que con mayor frecuencia se presentaron en nuestra población, en un estudio realizado en la población Quindiana se observó que tener unión marital puede disminuir 50\% la posibilidad del suicidio (16); de igual forma en la encuesta nacional de salud mental 2015 se encontró una mayor prevalencia del intento suicida en personas separadas, viudas y divorciadas al igual que se observa en el departamento de Risaralda y Caldas según los informes de dichos departamentos (14) (32).

La escolaridad predominante en quienes presentaron el intento de suicidio fue básica secundaria, similar a lo descrito en la encuesta nacional de salud mental 2015 en donde la mayor ideación suicida se presenta entre las personas con básica secundaria completa (5); la población con intentos previos de suicidio representó el 32,1\% para el boletín Colombiano de salud mental (10) y 30\% descrito en un artículo de la revista Colombiana de psiquiatría (12), comparado con la población de Armenia en la cual fue del 32,6\%.

En Colombia el $80 \%$ de la población que muere por suicidio tiene una enfermedad mental de base (12); dentro de estos trastornos mentales se destacan: trastorno de ansiedad, trastorno bipolar, esquizofrenia y depresión (13). En el estudio de suicidio y trastorno mental de la universidad Cooperativa de Colombia el $27 \%$ de la población con trastorno depresivo había tenido un intento de suicidio previo y para la población objeto de este estudio el mayor factor de riesgo fue el antecedente de algún trastorno psiquiátrico con $39 \%$ y antecedentes de depresión en un 34\% (20).

Según el boletín Colombiano de salud mental de conducta suicida del 2018 los principales factores de riesgo del intento de suicidio en relación a los encontrados en este estudio son: los conflictos de pareja $41,1 \%$ vs $38,4 \%$ para la población de Armenia, problemas económicos $11,5 \%$ vs $10,7 \%$ para la población de estudio, además mencionan los problemas escolares $(6,2 \%$ vs $0,84 \%$ ), el maltrato físico, psicológico o sexual $(5,8 \%$ vs $1,67 \%)$ los cuales no fueron relevantes para la población de este estudio (10). En cuanto al mecanismo de intento suicida utilizado más frecuente fue la intoxicación de $70,4 \%$ vs $70,9 \%$ como se encontró en un estudio realizado en Cali (33); seguido de arma cortopunzante.

Uno de los factores desencadenantes más importantes fueron enfermedad crónica dolorosa o discapacitante que concuerda con los resultados del estudio realizado en adultos de mediana edad y mayores en Estados unidos en el cual las condiciones de salud física están asociadas con un mayor riesgo de aparición de pensamientos y comportamientos suicidas (34).

Los factores que limitaron este estudio fueron los siguientes: El mal diligenciamiento de la ficha de notificación, el desconocimiento del instructivo de diligenciamiento por parte del personal salud, el sub registro del evento, los datos perdidos por actualizaciones constantes de las fichas de notificación, la falta de opción de respuesta en algunas de las variables; tales como: ninguno o sin información.

\section{CONCLUSIÓN}

En Armenia, el intento suicida es más frecuente: en mujeres, escolaridad básica o ninguna, estrato socioeconómico bajo. Dentro de los factores causales más importantes relacionados con intento de suicidio se encuentran los conflictos de pareja, la ideación suicida persistente y el antecedente de trastorno psiquiátrico. El método para el intento de suicidio más frecuentemente reportado es la intoxicación. La población sin pareja, recluidos en un centro psiquíatrico, con antecedentes de consumo de SPA y con antecedentes de enfermedad crónica dolorosa o discapacitantes se encontró relación para la población en la cual ha tenido intentos previos. Durante el año 2018 se incrementaron los casos notificados de intento de suicidio y para los 3 años de estudio se ve una mayor presentación para los periodos IX y XII. 
Se recomienda realizar un análisis de las actualizaciones que sean necesarias para la ficha de notificación de intento de suicidio al inicio del año para que una versión sea vigente por el año en curso y de esta forma no tener perdida de datos.

\section{AGRADECIMIENTOS}

Se agradece a la Secretaria de Salud de Armenia por facilitar la base de datos con la información de la ficha de notificación de intento de suicidio de SIVIGILA y a la Fundación Universitaria del Área Andina que nos brinda la infraestructura y los medios tecnológicos para llevar a cabo este proyecto.

\section{CONFLICTO DE INTERESES}

Los autores declaran no tener ningún tipo de conflicto de intereses en relación a las instituciones que intervinieron en la investigación.

\section{REFERENCIAS}

1. Rodríguez-Hernández JM, Rocha-Buelvas A, Mendieta-Izquierdo G, Hidalgo-Troya A. Riesgo de muerte por suicidio en población Colombiana 2000-2013. Ciênc saúde coletiva. noviembre de 2018; 23(11):3989-96. https://doi.org/10.1590/1413-812320182311.24812016

2. Rawat S, Rajkumari S, Joshi PC, Khan MA, Saraswathy KN. Who dies and who survives? Investigating the difference between suicide decedents and suicide attempters. Egypt J Forensic Sci. diciembre de 2019;9(1):10.

3. Organización Panamericana de la Salud. Mortalidad por suicidio en las Américas. Washington DC: Organización panamericana de la salud; 2014.

4. Rosenbaum Asarnow J, Berk M, Zhang L, Wang P, Tang L. Emergency Department Youth Patients With Suicidal Ideation or Attempts: Predicting Suicide Attempts Through 18 Months of FollowUp. Suicide Life Threat Behav. 2017;47(5):551-66. https://doi.org/10.1111/sltb.12309

5. López-Goñi JJ, Goñi-Sarriés A, Azcárate-Jiménez L, Sabater-Maestro P. Repetición de conductas suicidas en urgencias psiquiátricas en pacientes sin intentos previos, index y repetidores: un estudio prospectivo. Revista de Psiquiatría y Salud Mental. noviembre de 2018. https://doi.org/10.1016/j. rpsm.2018.07.003

6. Jiménez Q M, Hidalgo B J, Camargo S C, Dulce R B. El intento de suicidio en la población pediatica, una alarmante realidad. Rev Cienc Salud. 2014;12(1):59-83.

7. Kim B, Ahn J-H, Cha B, Chung Y-C, Ha TH, Hong Jeong S, et al. Characteristics of methods of suicide attempts in Korea: Korea National Suicide Survey (KNSS). Journal of Affective Disorders. 1 de diciembre de 2015;188:218-225. https://doi.org/10.1016/j.jad.2015.08.050

8. Andriessen K, Krysinska K, Hill NTM, Reifels L, Robinson J, Reavley N, et al. Effectiveness of interventions for people bereaved through suicide: a systematic review of controlled studies of grief, psychosocial and suicide-related outcomes. BMC Psychiatry. diciembre de 2019;19(1):49. https://doi.org/10.1186/s12888-019-2020-z

9. Dávila-Cervantes CA. Factores sociodemográficos asociados a la mortalidad por suicidios en México, 2012-2016. Universidad y Salud. diciembre de 2019;21(3):235-239. https://doi.org/10.22267/ rus. 192103.160

10. Boletin conducta suicida.pdf [Internet]. [citado 7 de marzo de 2020]. Disponible en: https://www. minsalud.gov.co/sites/rid/Lists/BibliotecaDigital/RIDE/VS/PP/ENT/boletin-conducta-suicida.pdf

11. Cardona Arango D, Medina-Pérez ÓA, Cardona Duque DV. Caracterización del suicidio en Colombia, 2000-2010. Revista Colombiana de Psiquiatría. julio de 2016;45(3):170-177. https://doi. org/10.1016/j.rcp.2015.10.002

12. Aparicio Castillo YA, Blandón Rodríguez AM, Chaves Torres N-M. Alta prevalencia de dos o más intentos de suicidio asociados con ideación suicida y enfermedad mental en Colombia en 2016. Revista Colombiana de Psiquiatría. agosto de 2018; 49(2): 96-101. https://doi.org/10.1016/j. rep.2018.07.001.

13. Cañon B SC, Castaño Castrillon JJ, Medina Jiménez N, Mosquera E KK, Quintero C CA, Varón C LL. Caracterización del intento suicida en pacientes ingresados a una institución de salud (Pereira, Colombia 2013-2014). MED UNAB 2017. 03/2017 de 2016;19 (3):192-202. 
14. Arenas A, Gómez-Restrepo C, Rondón M. Factores asociados a la conducta suicida en Colombia. Resultados de la Encuesta Nacional de Salud Mental 2015. Revista Colombiana de Psiquiatría. diciembre de 2016;45:68-75. https://doi.org/10.1016/j.rcp.2016.03.006

15. Ortíz IDG. Plan de conducta suicida concertacion intersectorial. Ministerio de salud y protección social. :42.

16. Medina-Pérez ÓA, Ospina-Sánchez SM, Cardona-Duque DV. Characterization of suicide in adolescents in the Quindio Department, Colombia, 1989 - 2013. Revista Habanera de Ciencias Médicas; 16:784-795. https://www.redalyc.org/articulo.oa?id=1804/180454487011

17. Parra-Uribe I, Blasco-Fontecilla H, Garcia-Parés G, Martínez-Naval L, Valero-Coppin O, CebriàMeca A, et al. Risk of re-attempts and suicide death after a suicide attempt: A survival analysis. BMC Psychiatry. 04 de 2017;17(1):163. https://doi.org/10.1186/s12888-017-1317-z

18. Organizacion mundial de la Salud. Plan de acción sobre salud mental 2013-2020. 2013.

19. De Beurs D, Fried EI, Wetherall K, Cleare S, O' Connor DB, Ferguson E, et al. Exploring the psychology of suicidal ideation: A theory driven network analysis. Behaviour Research and Therapy. 1 de septiembre de 2019;120:103419. https://doi.org/10.1016/j.brat.2019.103419

20. Bedoya Cardona EY, Montaño Villalba LE, Universidad Cooperativa de Colombia. Suicidio y Trastorno Mental. Rev. CES Psicol. 2016;(9)2:179-201.

21. Brüdern J, Stähli A, Gysin-Maillart A, Michel K, Reisch T, Jobes DA, et al. Reasons for living and dying in suicide attempters: a two-year prospective study. BMC Psychiatry. 20 de 2018;18(1):234. https://doi.org/10.1186/s12888-018-1814-8

22. Plan prevencion suicidio [Internet]. [citado 12 de marzo de 2020]. Disponible en: https://www. euskadi.eus/contenidos/informacion/estrategia_prevencion_suicidio/es_def/adjuntos/plan_prevencion_suicidio_cast-86-92.pdf

23. Exbrayat S, Coudrot C, Gourdon X, Gay A, Sevos J, Pellet J, et al. Effect of telephone follow-up on repeated suicide attempt in patients discharged from an emergency psychiatry department: a controlled study. BMC Psychiatry. diciembre de 2017;17(1):96. https://doi.org/10.1186/s12888017-1258-6

24. Gonzáles Portillo J, Franco Monroy DA, Marín Vasco JA, Restrepo Chacón S. Social Skills and Suicide Risk in Adolescents of an Education Institute of the City of Armenia (Quindío, Colombia). Revista Ciencias De La Salud. 8 de febrero de 2019;17(1):18-33. https://doi.org/10.12804/revistas. urosario.edu.co/revsalud/a.7611

25. Rueda-Jaimes GE, Castro-Rueda VA, Rangel-Martínez-Villalba AM, Corzo-Casasadiego JD, Moreno-Quijano C, Camacho PA. Validity of the Suicide Behaviors Questionnaire-Revised in patients with short-term suicide risk. The European Journal of Psychiatry. octubre de 2017;31(4):145-150. https://doi.org/10.1016/j.ejpsy.2017.09.002

26. García OEP, Bonilla HQ, Alfonso CA, Rodríguez AMB, Martínez Duran M. Protocolo de Vigilancia en Salud Pública Intento de suicidio. Instituto nacional de salud. 2014;19.

27. Guía de practica clínica en conducta suicida [Internet]. [citado 9 de marzo de 2020]. Disponible en: http://gpc.minsalud.gov.co/gpc_sites/Repositorio/Otros_conv/GPC_CSuicida/GPC_C_Suicida_Profesionales.pdf

28. Carmona J. La prevención del suicidio y la afirmación de la vida en una institución educativa: Un modelo de intervención psicosocial. Editorial El Manual Moderno Colombia S.A.S.; 2019. 236 p.

29. Grupo Centro de Referencia Nacional sobre Violencia. Forensis datos para la vida 2018 [Internet]. Instituto Nacional de Medicina Legal y Ciencias Forenses; Disponible en: https://www.medicinalegal.gov.co/cifras-estadisticas/forensis

30. Serrano Ruiz CP, Olave Chaves JA. Factores de riesgo asociados con la aparición de conductas suicidas en adolescentes. MedUNAB. 4 de agosto de 2017;20(2):139-47. https://doi. org/10.29375/01237047.2272

31. Castaño Castrillón JJ. Caracterización del intento suicida en pacientes que ingresaron al Instituto del Sistema Nervioso en la ciudad de Pereira, Risaralda, Colombia, 2013-2014. MedUNAB. 3 de agosto de 2017;19(3):192-202. https://doi.org/10.29375/01237047.2342

32. Cardona PNB, Torres ER, González CE, Portilla MB. Intento suicida y factores asociados en dos instituciones de Cali - Colombia.: Revista Ciencia y Cuidado. 1 de enero de 2019; 16(1): 19-31. https://doi.org/10.22463/17949831.1542

33. Dean-Boucher A, Robillard CL, Turner BJ. Chronic medical conditions and suicidal behaviors in a nationally representative sample of American adolescents. Soc Psychiatry Psychiatr Epidemiol. marzo de 2020;55(3):329-337. https://doi.org/10.1007/s00127-019-01770-2 